\title{
Le Comité international de la Croix-Rouge et les droits de l'homme
}

\author{
Schindler, Dietrich
}

\begin{abstract}
Au cours d'une même année, 1978, ont été Célébrés le 15e anniversaire de la naissance d'Henry Dunant, le 30e anniversaire de la Déclaration universelle des droits de l'homme et les 25 ans de la Convention européenne des droits de l'homme, tandis qu'entraient en vigueur la Convention américaine des droits de l'homme de 1969 et les Protocoles additionnels aux Conventions de Genève de 1977. La conjonction de ces événements mémorables invite à réfléchir aux rapports entre le droit international humanitaire et les droits de l'homme
\end{abstract}

DOI: https://doi.org/10.1017/s0035336100062055

Posted at the Zurich Open Repository and Archive, University of Zurich ZORA URL: https://doi.org/10.5167/uzh-154548

Journal Article

Published Version

Originally published at:

Schindler, Dietrich (1979). Le Comité international de la Croix-Rouge et les droits de l'homme. International Review of the Red Cross, 61(715):3-15.

DOI: https://doi.org/10.1017/s0035336100062055 


\section{LE COMITE INTERNATIONAL DE LA CROIX-ROUGE ET LES DROITS DE L'HOMME}

par D. Schindler

\section{Les voies distinctes de l'évolution du droit international humanitaire et des droits de l'homme}

Au cours d'une même année, 1978 , ont été célébrés le $150^{\circ}$ anniversaire de la naissance d'Henry Dunant, le $30^{\mathrm{e}}$ anniversaire de la Déclaration universelle des droits de l'homme et les 25 ans de la Convention européenne des droits de l'homme, tandis qu'entraient en vigueur la Convention américaine des droits de l'homme de 1969 et les Protocoles additionnels aux Conventions de Genève de 1977. La conjonction de ces événements mémorables invite à réfléchir aux rapports entre le droit international humanitaire et les droits de l'homme.

Après une longue période au cours de laquelle elles ont connu une évolution distincte, ces deux branches du droit international, depuis la deuxième guerre mondiale, se sont rapprochées de plus en plus, au point que leurs domaines d'application se recouvrent pour une bonne partie. Elles ont l'une et l'autre le même objectif: la protection de la personne humaine. En revanche, elles visent chacune des situations différentes et elles ont évolué de façon dissemblable. C'est de ce point qu'il sera tout d'abord question. 


\section{Le développement du droit international humanitaire}

Le droit international humanitaire, en tant que partie du droit de la guerre ou droit des conflits armés, tend à assurer une protection et un traitement humain aux personnes mises hors de combat ou qui ne participent pas aux hostilités.

Presque toutes les grandes civilisations de l'Antiquité et du Moyen Age ont connu des règles limitant le droit des belligérants à infliger des dommages à leur ennemi. Chez les Perses, les Grecs, les Romains, les Indiens, dans l'Islam et la Chine ancienne, en Afrique et dans l'Europe chrétienne, on peut relever des normes par l'effet desquelles certaines personnes sont protégées, tels les femmes, les enfants et les vieillards, les combattants désarmés et les prisonniers, tandis que sont interdites les attaques contre certains objets, comme les lieux sacrés, ou le recours à des moyens déloyaux, en particulier au poison.

Le droit de la guerre actuel s'est formé, sous l'influence surtout du christianisme et des règles de la chevalerie, à l'occasion des guerres que se sont livrées les Etats européens depuis l'apparition du système étatique européen moderne. Il a trouvé son expression dans des ordonnances ou règlements édictés par des Etats à l'intention de leurs armées, prescrivant à leurs troupes un comportement déterminé face à l'ennemi, et aussi dans des conventions bilatérales (cartels, capitulations, armistices), conclues entre les commandants militaires d'Etats ennemis et ayant pour objet les soins aux blessés ou l'échange des prisonniers de guerre. L'uniformité de ces réglementations suscita l'apparition d'un droit coutumier. Les écrits des grands auteurs du droit international, tels Grotius et Vattel, contribuèrent à consolider ces coutumes. Cependant, ce n'est qu'au $\mathrm{XIX}^{\mathrm{e}}$ siècle que, sous l'impression de guerres dans lesquelles combattaient désormais de grandes armées nationales, utilisant des armes nouvelles et plus meurtrières et faisant un nombre effrayant de blessés abandonnés sans aucun secours sur les champs de bataille, on s'attacha à élaborer un droit de la guerre sanctionné par des conventions multilatérales. Ce n'est pas par un effet du hasard que cette évolution se produisit à un moment où, sur le plan intérieur des Etats du monde occidental, des principes communs parvenaient à s'imposer en matière de droits de l'homme. La Convention de Genève de 1864 pour l'amélioration du sort des militaires blessés dans les armées en campagne marqua un progrès décisif $\mathrm{du}$ droit humanitaire international. Elle donna une expression claire à la notion de droits humains d'application générale, en faisant une obligation aux Etats contractants de traiter de la même manière leurs propres blessés et les blessés ennemis. 
Le Comité de Genève, qui avait été le promoteur de cette convention et qui devait poursuivre son action sous le nom de Comité international de la Croix-Rouge, s'attacha plus tard à développer le droit international humanitaire. Les décennies qui suivirent furent marquées par l'adoption de conventions visant à protéger encore d'autres catégories de personnes, à savoir, en 1899, une convention sur les membres des forces navales, en 1929, une autre sur les prisonniers de guerre, et, en 1949, une autre encore sur les personnes civiles. De plus, les conventions déjà adoptées furent révisées en 1906, 1929 et 1949. Enfin, en 1977, furent adoptés les deux Protocoles additionnels complétant les quatre conventions de 1949.

Alors que les Conventions de Genève ne traitent toutes que de la protection de personnes tombées aux mains de l'ennemi (blessés, malades, prisonniers de guerre, personnes civiles), les Conventions de La Haye de 1899 et de 1907 visent en premier lieu à régler la conduite des opérations militaires. Elles interdisent aux Etats belligérants d'attaquer certaines personnes et certains objectifs et de recourir à certains moyens et certaines méthodes de guerre. Une partie de ces règles ont été confirmées et développées par les deux Protocoles additionnels de 1977. Bien que, en dernière analyse, les Conventions de La Haye aient pour objet de protéger des êtres humains, les préoccupations humanitaires se manifestent avec plus de force dans les Conventions de Genève, car celles-ci traitent directement du sort des êtres humains affectés par la guerre.

Parallèlement au développement du droit humanitaire international, le Comité international de la Croix-Rouge a aussi contribué de diverses autres manières à la protection des victimes de conflits armés et, de ce fait, à la défense des droits de l'homme. Les Conventions de Genève elles-mêmes lui confient la tâche de participer à leur propre mise en œuvre et de surveiller leur application, ce qu'il fait notamment en visitant les prisonniers de guerre et les internés civils. De plus, le Comité a entrepris les actions de secours les plus diverses, en faveur non seulement des personnes frappées par une guerre (internationale ou interne), mais encore en faveur des victimes de troubles intérieurs, voire de simples tensions. Dans l'optique de la protection des droits de l'homme, une importance particulière s'attache au fait que les délégués du Comité ont $\mathrm{pu}$, depuis la fin de la deuxième guerre mondiale et en dehors de conflits armés, visiter, dans plus de 70 pays, quelque 300.000 détenus politiques, que ne protégeait aucune convention. De ce fait, la protection a pu être étendue à une catégorie de personnes qui se trouvent dans une situation analogue à celle des prisonniers de guerre ou des internés civils, mais en faveur de qui les Etats ne seraient guère disposés à conclure une convention, car il s'agit, dans la plupart des cas, de leurs propres ressortissants. 


\section{Le développement des droits de l'homme}

Les droits de l'homme et le droit de la guerre se sont développés de manières différentes et totalement distinctes, bien que leurs racines spirituelles soient partiellement les mêmes et que, à partir du XIX ${ }^{\mathbf{e}}$ siècle, on puisse observer un certain parallélisme dans l'évolution de ces deux branches du droit. Les premiers énoncés des droits de l'homme figurent dans les déclarations adoptées à leur sujet par certains Etats américains

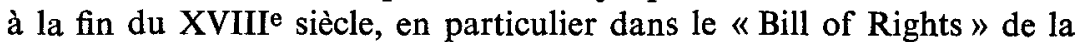
Virginie en 1776, comme dans la Déclaration française des droits de l'homme et du citoyen de 1789. Ces proclamations étaient d'ailleurs l'aboutissement d'un assez long processus. L'histoire constitutionnelle anglaise est à cet égard importante. Les Anglais parvinrent à arracher au roi et au gouvernement certains droits que vinrent sanctionner diverses chartes, tels la "Petition of Rights 》 de 1628, le « Habeas Corpus Act 》 de 1679, le "Bill of Rights» de 1689. Ces droits n'étaient pas opposables aux compétences du Parlement et n'étaient pas ressentis comme des droits fondamentaux ou des droits de l'homme, au sens où nous les entendons aujourd'hui, mais la plupart d'entre eux n'en ont pas moins trouvé leur place dans les déclarations des droits de l'homme de la période révolutionnaire, une portée plus large leur étant ainsi attribuée. Ces déclarations sont d'ailleurs l'aboutissement d'une longue évolution historique des idées. Celle-ci fut amorcée dès l'Antiquité par le stoïcisme, qui a été le premier à insister sur l'égalité de tous les êtres humains, surmontant ainsi l'isolement réciproque des peuples et le refus de tout statut juridique aux étrangers. Cette philosophie trouva un appui dans la création de l'empire mondial d'Alexandre, puis dans l'institution de l'empire romain. Elle se conjugua plus tard avec la doctrine chrétienne, selon laquelle l'homme est fait à l'image de Dieu et tous les êtres humains sont égaux. Cette notion de l'égalité des hommes a imprégné le droit naturel du Moyen Age et du début des temps modernes. Finalement, elle déboucha dans l'enseignement des philosophes du siècle des lumières, sur lequel sont fondées les déclarations américaine et française des droits de l'homme.

$\mathrm{Au} \mathrm{XIX}^{\mathrm{e}}$ siècle, les déclarations de droits fondamentaux trouvent de plus en plus fréquemment une place dans les constitutions nationales et, à l'heure actuelle, le droit constitutionnel de presque tous les Etats comporte des garanties de cet ordre. Mais de telles sanctions firent défaut sur le plan international jusqu'à la deuxième guerre mondiale, exception faite de conventions internationales sur quelques aspects particuliers des droits de l'homme, comme la prohibition de l'esclavage ou la protection des minorités. 
De tout temps, ces garanties des droits de l'homme ont visé les rapports entre l'Etat en cause et ses propres ressortissants et se sont rapportées au temps de paix. Elles n'ont jamais pris en considération le traitement des personnes ennemies en temps de guerre. Cette dissociation des droits de l'homme et du droit de la guerre persista même lorsque, après la deuxième guerre mondiale, des conventions de droit international relatives aux droits de l'homme furent conclues. Celles-ci aussi règlent au premier chef les rapports entre les Etats et leurs propres citoyens. Elles ont été conclues parce qu'on s'était rendu compte que le respect des droits de l'homme sur le plan intérieur des Etats est une condition du maintien de la paix. C'est ainsi que le Secrétaire général des Nations Unies, dans son premier rapport sur "Le respect des droits de l'homme en période de conflit armé », daté de 1969 (A.7720), déclare, au paragraphe 16 , que « la deuxième guerre mondiale a montré de façon concluante la relation étroite qui existe entre l'attitude révoltante d'un gouvernement à l'égard de ses propres ressortissants et l'agression qu'il perpètre contre d'autres nations et, par conséquent, entre le respect des droits de l'homme et le maintien de la paix. " Cette appartenance des droits de l'homme au domaine interne est la raison pour laquelle les conventions qui s'y rapportent ont été jusqu'ici moins largement ratifiées que les Conventions de Genève. Au 31 décembre 1978, 52 Etats étaient parties au Pacte des Nations Unies sur les droits civils et politiques (et 54 au Pacte sur les droits économiques, sociaux et culturels), alors que 145 Etats avaient souscrit aux Conventions de Genève.

\section{L'apparition, depuis la deuxième guerre mondiale, de connexions entre le droit international humanitaire et les droits de l'homme}

Au sein des Nations Unies, on a estimé tout d'abord que, si l'Organisation s'occupait du droit de la guerre, l'opinion douterait de sa capacité de maintenir la paix. En conséquence, lors de sa première session tenue en 1949, la Commission du droit international des Nations Unies décida que le droit de la guerre ne figurerait pas au nombre des objets dont elle aurait à traiter. La Déclaration universelle de 1948 laisse totalement à l'écart la question du respect des droits de l'homme dans les conflits armés. Parallèlement, lors de l'élaboration des Conventions de Genève de 1949, il n'a guère été question des droits de l'homme.

Toutefois, il s'est établi entre les Conventions de Genève et les conventions des droits de l'homme des liaisons qui mettent ces deux branches 
$\mathrm{du}$ droit international en rapport l'une avec l'autre sans que, le plus souvent, on en ait eu l'intention. En effet, d'un côté, on peut déceler dans les Conventions de Genève de 1949 une tendance à considérer leurs stipulations comme établissant non plus seulement des obligations qui grèvent les Etats contractants mais aussi des droits individuels dont les personnes protégées jouissent. C'est ainsi qu'il est prévu dans chacune des quatre conventions que les personnes protégées ne peuvent pas renoncer aux droits que celles-ci leur accordent (article 7 des trois premières conventions, article 8 de la quatrième). De surcroît, l'article 3 , commun aux quatre conventions, impose aux parties, dans les conflits de caractère non international, le respect de règles minimales d'humanité; il réglemente ainsi les rapports entre les Etats et leurs propres ressortissants et empiète, par conséquent, sur le domaine traditionnel des droits de l'homme. On reconnaîtra également l'influence du mouvement des droits de l'homme dans le fait que, au cours des années cinquante, on a commencé à parler de "droit international humanitaire " à propos des Conventions de Genève, cette désignation par la suite appliquée dans une certaine mesure à l'ensemble du droit de la guerre ou droit des conflits armés.

D'un autre côté, dans les conventions des droits de l'homme figurent des dispositions relatives à leur application en temps de guerre. La Convention européenne des droits de l'homme, de 1950, prévoit à son article 15 que, en cas de guerre ou en cas d'autre danger public menaçant la vie de la nation, il peut être dérogé aux droits sanctionnés par la convention, à l'exception de quatre droits, maintenus même en de tels cas, qui constituent un noyau irréductible. Des dispositions analogues se retrouvent à l'article 4 du Pacte des Nations Unies relatif aux droits civils et politiques et à l'article 27 de la Convention américaine des droits de l'homme. Ainsi donc, en principe, les conventions sur les droits de l'homme sont également applicables dans les conflits armés. Lorsqu'un conflit ne menace pas la vie de la nation, ce qui peut être le cas lors d'interventions militaires limitées d'un Etat sur le territoire d'un autre Etat, les conventions des droits de l'homme sont même applicables dans toute leur étendue, parallèlement au droit international humanitaire.

Longtemps, on n'a guère pris garde à ces connexions entre ces deux branches du droit international. Ce n'est que vers la fin des années soixante qu'on en est devenu conscient. L'occasion en fut donnée par les conflits armés de cette époque, comme les guerres de libération en Afrique, le conflit du Proche-Orient, les conflits du Nigeria et du Viet-Nam, qui ont présenté simultanément des aspects relevant du droit de la guerre et des droits de l'homme. La Conférence sur les droits de l'homme, convoquée 
par les Nations Unies en 1968 à Téhéran, a jeté officiellement un pont entre les droits de l'homme et le droit international humanitaire. En effet, par sa résolution XXIII du 12 mai 1968 concernant le « respect des droits de l'homme en période de conflit armé ", la Conférence demandait une meilleure application des conventions sur les conflits armés existantes et la conclusion de nouveaux accords. En même temps, cette résolution conduisait les Nations Unies à s'occuper désormais du droit international humanitaire. Les rapports annuels du Secrétaire général et les résolutions annuelles de l'Assemblée générale témoignent de cette préoccupation. Il a fallu cette initiative de la Conférence de Téhéran pour que les Etats se montrent disposés à compléter les Conventions de Genève, alors que le « Projet de règles limitant les risques courus par la population civile en temps de guerre », présenté par le Comité international de la CroixRouge en 1956, n'avait pas suscité de telles dispositions.

Cette impulsion provenant de la sphère des droits de l'homme a eu des effets sur la teneur des deux Protocoles additionnels de 1977. Plusieurs de leurs dispositions s'inspirent directement de celles du Pacte des Nations Unies sur les droits civils et politiques. Tel est le cas de l'article $75 \mathrm{du}$ Protocole I (Garanties fondamentales) et de l'article $6 \mathrm{du}$ Protocole II (Poursuites pénales).

La convergence du droit humanitaire international et des droits de l'homme contribue à montrer que la guerre et la paix, les guerres civiles et les conflits internationaux, le droit international et le droit interne s'intègrent de plus en plus étroitement. Le droit de la guerre et le droit de la paix, le droit international et le droit interne, dont les domaines d'application étaient à l'origine clairement séparés, sont souvent aujourd'hui applicables simultanément et parallèlement. Ainsi, les Conventions de Genève et les conventions des droits de l'homme peuvent souvent s'appliquer de manière cumulative.

\section{Maintien de conventions séparées pour les droits de l'homme et pour le droit international humanitaire}

Il y a chevauchement des domaines des droits de l'homme et du droit international humanitaire. Est-ce à dire que les conventions sur les droits de l'homme, lorsqu'elles auront été universellement ratifiées, rendront superflu le droit de Genève et de La Haye ? Evidemment non. Pour le démontrer, nous distinguerons entre deux éléments: le degré de concordance quant au fond des deux groupes de conventions, l'efficacité des 
mécanismes de contrôle et des sanctions prévus dans les deux groupes de conventions.

\section{La concordance des règles quant au fond}

Quant au fond, il apparaît que les Conventions de Genève accordent aux victimes des conflits armés une protection supérieure à celle des conventions des droits de l'homme, parce qu'elles sont mieux adaptées aux circonstances particulières de tels conflits.

Les dispositions des diverses conventions des droits de l'homme ont été élaborées, pour la plupart, sans tenir compte des conditions propres aux conflits armés. Cela apparaît par exemple dans le fait que le Pacte des Nations Unies sur les droits civils et politiques ne vise que les personnes qui se trouvent sur le territoire d'un Etat co-contractant (article 2, 1 er alinéa) et non les personnes qui sont à l'extérieur de ce territoire, extension qui serait indispensable pour le cas d'un conflit armé international. Il est symptomatique également que la Convention européenne des droits de l'homme, à son article 5, omette de mentionner, parmi les cas admissibles de privations de liberté, la capture de prisonniers de guerre et l'internement pour raisons de sécurité. De même, les prescriptions des conventions des droits de l'homme relatives aux droits individuels et à leurs limitations ne seraient pas suffisantes dans un conflit armé. Les exemples ci-après suffiront à montrer que les Conventions de Genève sont en mesure d'offrir aux personnes protégées une protection plus étendue et mieux adaptée aux circonstances d'un conflit armé que les conventions des droits de l'homme.

Le droit à la vie est l'un des premiers droits consacrés par ces dernières conventions (article 6 du Pacte des Nations Unies; article 2 de la Convention européenne; article 4 de la Convention américaine), avec certaines exceptions telles que la peine de mort, l'homicide en cas de légitime défense ou en état de nécessité. Le conflit armé appelle une définition plus précise du droit à la vie, puisque tuer les membres du personnel militaire ennemi est un acte légitime. C'est pourquoi les Conventions de Genève (et leurs Protocoles additionnels) et les Conventions de La Haye précisent qu'il est interdit d'attenter à la vie des personnes qu'elles entendent protéger (blessés, malades, prisonniers de guerre, personnes civiles); elles interdisent de même de tuer par trahison des personnes ennemies, ainsi que celles qui ont mis bas les armes, se sont rendues ou sont sans défense. Tombent également sous cette interdiction la mise à mort de personnes qui sautent en parachute d'un avion en perdition, les attaques menées sans discrimination, toute action visant à affamer la population civile, 
la destruction de biens et d'installations indispensables à la survie de la population civile, etc. Le droit à la vie dans un conflit armé n'aurait pas été suffisamment sanctionné en l'absence de telles précisions.

Les conventions sur les droits de l'homme garantissent ensuite le droit à la liberté, là encore sous réserve d'exceptions déterminées, en particulier la privation de liberté découlant d'une infraction pénale. Elles prohibent de même l'esclavage et le travail forcé, ce dernier également sous certaines réserves (articles 8 et 9 du Pacte des Nations Unies; articles 4 et 5 de la Convention européenne; articles 6 et 7 de la Convention américaine). Mais ces droits sont aussi précisés dans les conventions humanitaires applicables en cas de conflit armé. On y trouve notamment la prohibition de la prise d'otages et de la déportation des personnes civiles. La privation de liberté des prisonniers de guerre, la rétention du personnel sanitaire tombé aux mains de l'ennemi, l'internement de personnes civiles ennemies, le droit d'imposer un travail aux prisonniers de guerre et aux personnes civiles ennemies et d'autres questions connexes font aussi l'objet de stipulations détaillées.

Il serait aisé de démontrer par d'autres exemples que les conventions humanitaires applicables en cas de conflit armé contiennent des dispositions de protection des individus plus étendues et plus précises que les conventions sur les droits de l'homme. Cela vaut également, d'une manière générale, pour les conflits non internationaux. Certes, l'article 3 commun à ces conventions ne va guère plus loin que le minimum irréductible des conventions sur les droits de l'homme, mais le Protocole additionnel II de 1977 institue de nombreux droits plus étendus.

Le droit des conflits armés n'a cependant pas pour seul rôle d'adapter certains droits de l'homme à la situation particulière des conflits armés et de les concrétiser dans ce cadre. Il établit en fait des règles qui vont au-delà du domaine des droits de l'homme, tout comme les conventions sur les droits de l'homme contiennent des dispositions qui sont sans pertinence en cas de conflit armé. En d'autres termes, le droit des conflits armés et les droits de l'homme ne se recouvrent que partiellement. Le premier régit, par exemple, le droit de participer aux combats, la conduite des opérations militaires, la conduite de la guerre économique, en particulier dans la guerre maritime, ainsi que les relations entre Etats belligérants et Etats neutres. De telles questions n'auraient pas leur place dans les conventions sur les droits de l'homme. Ces dernières, pour leur part, établissent des droits qui n'ont pas de rôle à jouer dans un conflit armé. Tel est le cas des droits politiques ou de certaines libertés de portée politique, comme la liberté de la presse, la liberté d'expression, la liberté de réunion et d'association. 


\section{Les mécanismes de contrôle et les sanctions}

Si le droit humanitaire international et les conventions sur les droits. de l'homme coïncident en partie dans leur teneur, les mécanismes de contrôle et les sanctions sont réglés de façons différentes dans ces deux groupes de conventions.

Les Conventions de Genève sont appliquées avec le concours et sous le contrôle de puissances protectrices et du Comité international de la Croix-Rouge. Leurs représentants ont le droit de se rendre dans tous les lieux où se trouvent des prisonniers de guerre ou des personnes civiles protégées et de s'entretenir avec les prisonniers ou internés sans témoins. Leurs rapports et leurs recommandations restent confidentiels, ce qui permet aux Etats d'accepter plus facilement les visites. D'une manière générale, il est donné suite aux recommandations et réclamations ainsi formulées.

$\mathrm{Si}$, dans un conflit international, les parties sont tenues d'admettre des organes de contrôle, dans un conflit de caractère non international, un organisme humanitaire impartial, tel que le Comité international de la Croix-Rouge, peut uniquement offrir ses services aux parties en cause. En revanche, le CICR a le droit, dans un conflit international comme dans un conflit de caractère non international, de prendre de son propre mouvement des initiatives, en vue de protéger des personnes affectées par le conflit. Il fait usage de ce droit dans tous les conflits armés, que ce soit pour apporter des secours matériels, pour procéder à des échanges de prisonniers de guerre ou pour visiter des personnes privées de leur liberté.

Pour ce qui est de la répression des violations, le droit de la guerre a ceci de particulier que ses dispositions obligent non seulement les Etats mais aussi et directement les individus. Les Etats peuvent donc punir les personnes responsables de violations en invoquant directement le droit international. Les Conventions de Genève et le Protocole additionnel I de 1977 imposent aux parties contractantes l'obligation d'édicter des sanctions pénales visant à réprimer les infractions graves et à en poursuivre les auteurs. Cette garantie du droit des conflits armés par une répression pénale trouve son origine et sa justification dans le fait qu'il incombe avant tout aux membres des forces armées d'appliquer le droit de la guerre, une sanction pénale apparaissant dès lors comme le moyen le plus adéquat pour réprimer des actes contraires au droit dont ces militaires se rendraient coupables. Il y a, sur ce point, une différence essentielle entre le droit de la guerre et le système des droits de l'homme. En cas de violation de ces derniers droits, ce sont en première analyse 
les victimes qui doivent porter plainte auprès des autorités compétentes nationales et, éventuellement, internationales. En droit humanitaire international, le dépôt de plaintes par des personnes lésées dans leurs droits n'entre en règle générale pas en ligne de compte et cela pour deux raisons: normalement, les actes de soldats ne peuvent pas faire l'objet d'une procédure de plainte et le droit humanitaire international protège surtout des êtres humains désarmés et sans défense qui, le plus souvent, n'ont pas accès aux procédures nationales ou internationales de défense du droit. L'application des conventions humanitaires est donc mieux assurée par l'intervention d'un organisme neutre, pouvant agir de son propre chef, de même que par les sanctions pénales supplémentaires découlant de ces conventions.

Les conventions sur les droits de l'homme, pour leur part, prévoient que soit les Etats contractants soit les particuliers dont les droits ont été lésés peuvent porter plainte contre l'Etat fautif. La Convention européenne stipule que les Etats contractants ont sans restriction faculté d'intenter une requête, mais que les individus n'ont le même droit que si l'Etat en cause a émis une déclaration particulière à cet effet. A l'inverse, la Convention américaine ouvre cette procédure aux Etats, à condition que ceux-ci aient publié une déclaration à cet effet, tandis que les individus peuvent agir en toutes circonstances. Dans le cas du Pacte des Nations Unies, la procédure de requête (on parle à ce propos de «communication $»)$ est plus restreinte. Les communications étatiques ne sont admises que si l'Etat demandeur et l'Etat défendeur ont fait une déclaration spéciale, qui peut être retirée à tout moment. Les communications individuelles ne sont autorisées que si elles visent un Etat qui a ratifié le protocole facultatif annexé au Pacte, ce protocole pouvant être dénoncé avec un délai de trois mois.

En raison de son caractère facultatif et de la brièveté du délai de dénonciation, la procédure instituée par le Pacte des Nations Unies n'a qu'une valeur réduite en cas de conflit armé. Les procédures de la Convention européenne et de la Convention américaine, qui ne sont sujettes à aucune suspension en cas d'urgence, peuvent jouer un rôle plus important en cas de conflit, à condition que les péripéties de la guerre ne paralysent pas les organes compétents. Elles peuvent, notamment, prendre une grande valeur dans un conflit de caractère non international, cas pour lequel l'institution de la puissance protectrice est inconnue et où le CICR n'est admis qu'à offrir ses services. Les procédures prévues par les conventions des droits de l'homme sont lourdes - elles peuvent se prolonger -sur plusieurs années - mais la publicité qui s'y rattache peut avoir un effet préventif considérable. 
Quant aux mécanismes de contrôle des deux types de conventions, ils peuvent, sans grandes difficultés, être mis en action d'une manière cumulative, du fait qu'en règle générale leurs modalités sont tout à fait différentes. Le contrôle exercé par le CICR ou la puissance protectrice a des effets plus rapides et plus directs que celui qui résulte du déroulement des procédures prévues par les conventions des droits de l'homme, de telle sorte que celles-ci peuvent, selon les circonstances, en devenir superflues. On peut cependant songer à des situations où les organes des droits de l'homme peuvent agir plus efficacement que le CICR, tout particulièrement lorsqu'ils sont habilités à intervenir de leur propre initiative, comme c'est le cas de la Commission interaméricaine des droits de l'homme. Lors de la guerre civile qui s'est déroulée en 1965 en République dominicaine, cette commission, qui existait déjà à l'époque, a déployé une grande activité de visite des personnes incarcérées. Elle le fit d'entente avec les délégués du CICR qui se trouvaient sur place. Les deux organisations se sont utilement réparti les tâches à accomplir et se sont complétées dans leurs activités.

\section{Conclusions}

Depuis la deuxième guerre mondiale, l'idée s'est propagée qu'il était nécessaire d'instaurer une garantie internationale des droits de l'homme. Ce mouvement n'a pas seulement conduit à la conclusion des conventions des droits de l'homme, il a donné aussi un vigoureux essor au droit humanitaire. Sans l'impulsion provenant des droits de l'homme, l'adoption des deux Protocoles additionnels de 1977 aux Conventions de Genève n'aurait guère été possible. C'est donc à juste titre que l'on veut voir s'établir un lien étroit entre ces deux branches du droit international et que l'on tient leur coordination pour nécessaire. Il n'en reste pas moins opportun que les droits de l'homme et le droit humanitaire fassent l'objet de traités distincts. Les circonstances d'un conflit armé exigent des mesures plus précises, et en partie différentes de celles qui valent pour le temps de paix. De plus, il faut que les dispositions de droit humanitaire soient complétées par des règles relatives à la conduite de la guerre, qui sont étrangères au domaine des droits de l'homme et doivent par conséquent faire l'objet d'un traitement séparé.

Il est, en outre, souhaitable que le contrôle de l'application des conventions des droits de l'homme et des conventions humanitaires soit exercé par des organes différents. L'action d'intermédiaire de la puissance protectrice ou du CICR, prenant la forme des visites de lieux de détention et 
de la communication de rapports confidentiels, donne, en temps de conflit armé, plus de résultats que des plaintes formelles. En règle générale, il ne peut réellement être recouru à une procédure de plainte qu'en temps de paix, lorsque les individus accèdent sans obstacle aux organes nationaux ou internationaux et lorsque l'autorité judiciaire compétente a la possibilité de procéder à des enquêtes sur les violations alléguées, d'organiser des procédures de conciliation des parties et de prononcer des arrêts fondés en droit. Lorsque, dans des cas particuliers, les procédures propres à l'un et à l'autre type de conventions peuvent être suivies concurremment, ce concours ne présente pas d'inconvénient. Il ne peut en résulter qu'un renforcement de la protection des personnes intéressées.

Une réglementation du droit des conflits armés distincte de celle des droits de l'homme est à préconiser pour une raison de plus, à savoir que les conventions humanitaires sont plus largement acceptées que les conventions sur les droits de l'homme. Le droit des conflits armés vise des questions traitées depuis longtemps par le droit international. Les parties ont le plus souvent un intérêt réciproque à le voir respecté. Les droits de l'homme, en revanche, ont été considérés jusqu'à une date récente comme relevant du domaine interne des Etats, et tel est encore le cas aujourd'hui dans une large mesure. Ils sont soumis plus fortement que le droit des conflits armés aux effets de la diversité des conceptions de l'Etat et des oppositions idéologiques. L'adoption des deux Protocoles additionnels aux Conventions de Genève de 1977 montre bien qu'une réglementation distincte du droit des conflits armés répond d'ailleurs à la volonté des Etats.

Pour toutes ces raisons, il continuera d'être possible au Comité international de la Croix-Rouge d'apporter une contribution essentielle à la réalisation des droits de l'homme, du fait qu'il s'emploie à assurer l'application des conventions humanitaires existantes ainsi que leur développement et qu'il prend, indépendamment de ces conventions, des mesures visant à protéger les personnes affectées par des conflits armés ou des troubles intérieurs.

\author{
Dietrich SCHINDLER \\ Professeur à la Faculté de droit \\ de l'Université de Zurich \\ Membre de la Commission juridique \\ du CICR
}

